\title{
Congestion Control in Asynchronous Transfer Mode (ATM) Network
}

\author{
Onyejegbu Laeticia Nneka \\ Department of Computer Science \\ University of Port-Harcourt \\ Port-Harcourt, Nigeria
}

\author{
Okafor Nkiru Rita \\ Department of computer Science \\ University of Port-Harcourt \\ Port-Harcourt, Nigeria
}

\begin{abstract}
Congestion has posed a big problem in (ATM) network. Every network user encounters congestion issues. In terms of network, congestion is unpredictable; it can be caused by traffic or too many data that cluster together in the system. In telecommunication, congestion occurs when more users access the network; during the peak period when the control channel is congested as such call cannot be establishment between the sender and receiver. In this paper, fuzzy logic and the proposed parameter called call completion success rate was used to regulate the network instability in Asynchronous Transfer Mode. It was observed that the call completion success rate enabled users to make calls without distortion of network. Comparison was made between fuzzy logic and call completion success rate. It was observed that, congestion load environment 4 has a value for mean bit rate as 1.4 , mean burst as 1.4 , state of network as 0.94 and retainability 0.9222 the last two are positive. The output signal to the service rate is 0.99 also positive. This implies that, the service rate should be free for calls to flow. The simulation results, shows ways of controlling congestion in ATM Network. The methodology adopted is Object Oriented Analysis and Design Methodology. It was implemented using Java Programming language and Matlab.
\end{abstract}

\section{General Terms}

Leaky bucket Algorithm, Congestion, Connection Admission Control, network capacity, fuzzy policer, Congestion control

\section{Keywords}

Asynchronous Transfer Mode, Network, Call Retainability, Fuzzy Logic, Call Completion Success Rate.

\section{INTRODUCTION}

Asynchronous Transfer Mode (ATM) network plays important role in performance of the network. (ATM) helps network users to reach the agreement when the connection is accepted, and also guarantee the quality of service [1]. Without traffic and congestion control techniques, traffic from user nodes can exceed the capacity of the network, causing memory buffers of ATM switches to overflow and leading to data losses[2].[3] (ATM) supports transport rate which distribute data to multiple users of network in a related manner. Congestion occurs when network performance fall off dramatically [4]. [5], described congestion as when no free path can be provided for an offered call as a result of network blockage, at that point, network user cannot obtain a connection immediately. Many times, calls are difficult to connect or when a data is sent, it takes times to deliver. Congestion in the network system can frustrate daily activities, the number of calls congested per day is more than call received meaning that, the telecommunication system are losing in terms of resources but when more calls get connected, it will also increase the resources. When a system is unable to transfer data or connect call, it implies that the network state is congested. Congestion leads to delay, mostly when too many data are transmitted at the same time or when many calls are queued in a transmission channel [6]. Congestion control, is a process in which networks adjust the amount of input data, so that the quality of service is not degraded and simultaneously trying to utilize the network resource efficiency [7]. According to [9] the link between congestion control and Asynchronous Transfer Mode is that congestion is essential for Asynchronous Transfer Mode network that guarantees both normal and exceptional conditions, to guarantee quality of service for all admitted connection and to improve network efficiency through a statistical multiplexing process . The problem of congestion in Asynchronous Transfer Mode, mostly in telecommunication is unavailability of the network to the subscriber at a time of making a call, queuing delay that is a delay in transmission of call and packet loss[14]. These problems need to be addressed for better performance of the network and to maintain quality of service. To control congestion problem, fuzzy logic was implemented because of its simplicity. Fuzzy logic is a logical system that has an extension of multi-valued logic. It was introduced by Zadeh and firstly applied by Mamdani to design control systems [10]. A fuzzy logic performs traffic and congestion control function and the idea of fuzzy logic is to convert, and then translate all the expertise of the human operator and incorporate them into a controller [11]. In the proposed system, a system was developed for controlling congestion in Asynchronous Transfer Mode. A multi-user simulation was done in congestion control system for Mobile Transfer Network that helped in reducing congestion in (ATM) network. This was achieved using call completion success rate. Congestion control has been a challenge, and therefore new control systems need to be implemented.

\section{REVIEW OF RELATED WORK}

[12], discussed the use of fuzzy logic based and neuro-fuzzy based mechanism for controlling the flow rate over Asynchronous Transfer Mode service which utilized a specific method of Available Bit rate (ABR) service of Asynchronous Transfer Mode network. They compared the performance of both methods in various cases. They showed that neuro-fuzzy based mechanism for controlling Availability Bit Rate in Asynchronous Transfer Mode networks outperformed the Fuzzy mechanism by simulation. [8], describe the rate feedback data flow control and proposed a fuzzy logic that ensures stability performance. It was shown from the simulation result that proposed controller is robust to uncertainty caused by communication channel delay. [11], proposed the use of fuzzy logic prediction on Connection, Admission Control, and congestion control in network that has high speed. Firstly, fuzzy logic predictor was applied to 
congestion control in which Available Bit Rate queue was estimated one round trip time in advance. It was shown, that fuzzy logic scheme decreased the convergence time and the overall buffer needs, to compare to traditional schemes. Secondly, they modeled fuzzy logic prediction on Connection Admission Control. By using simulation, it was shown that fuzzy logic prediction enhanced, the efficiency of both conventional as a well as fuzzy based Connection Admission Control. [7], discussed the fuzzy logic based congestion control in Asynchronous Transfer Mode networks. Their simulation result shows that fuzzy Explicit Rate Marking showed a robust performance under severe loading condition and ensures fair split of bandwidth for all virtual channel regardless of the number of hop [7].

\section{METHODOLOGY}

For every suitable software, there is a need for developing methodology which contributes to the success of developed application. The Object-Oriented, Analysis, and Design Methodology was adopted in the analysis and design of a network congestion control because it was best for implementation of the design.

\section{ARCHITETURAL DESIGN OF FUZZY LOGIC BASED TRAFFIC CONTROLLER}

When the network user dials a number, cell arrival detector, detect the incoming calls and send signal to fuzzy policer concerning the transmission. It does not connect the call immediately and it has some maximum capacity of calls to attain. Fuzzy policer, alert fuzzy congestion controller whenever congestion occurs. The fuzzy policer is a threeinput, nine-rule, and one -output system. The information from the fuzzy policer will decide whether to pass or drop the call depending on the state of the network. Whenever a call is passed, it is stored in the buffer and the result is outputted [13].

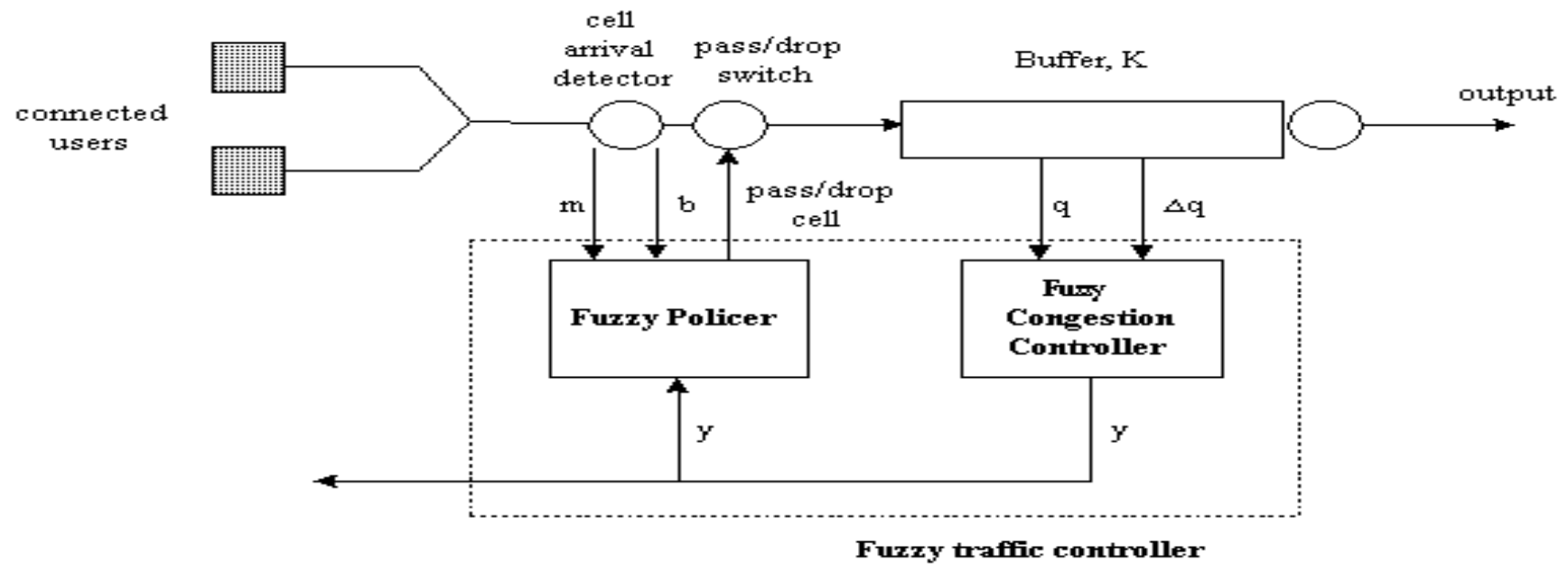

Figure:1 Architecture of the existing system (Weng el al, 1999)

\section{PROPOSED SYSTEM}

Call completion success rate is proposed as a parameter indicator that send signal whenever the system is experiencing congestion. Whenever the incoming calls are $75 \%$, the call completion success rate will send signals to fuzzy policer to retained $30 \%$ and allow $45 \%$ to pass. When network state is free, call completion success rate will also, send a signal to fuzzy policer to allow the remaining $30 \%$ and also increase the number of calls. It makes use of expression that intelligently makes accurate decisions on whether to pass or drop calls. When the user of the network send a call, the job of call completion success rate, is to detect when the system is congested, it ensure that the calls are completed successfully.

The call completion success rates guarantee network quality that can drive the network state. It helps to control congestion in the system by continuously evaluating the network state and, also helps the users to make call successfully without the distortion of the network. Fuzzy logic was improved to control Asynchronous Transfer Mode (ATM) Network using call completion success rate. The proposed system ensures congestion free and improve retain ability to the users of the network.

The indicator is derived using the expression:

CCS $=\frac{\text { Total Number of Call Attempts }}{\text { Total Number of Completed Call }} * 100 \%$

\section{Where CCS is Call Completion Success rate}

The proposed system has four-input, twenty-four rules, and one-output system. The additional parameter, Call Completion Success Rate, increased the existing rule to twenty-four rules to improve the performance of fuzzy policer (FP). 


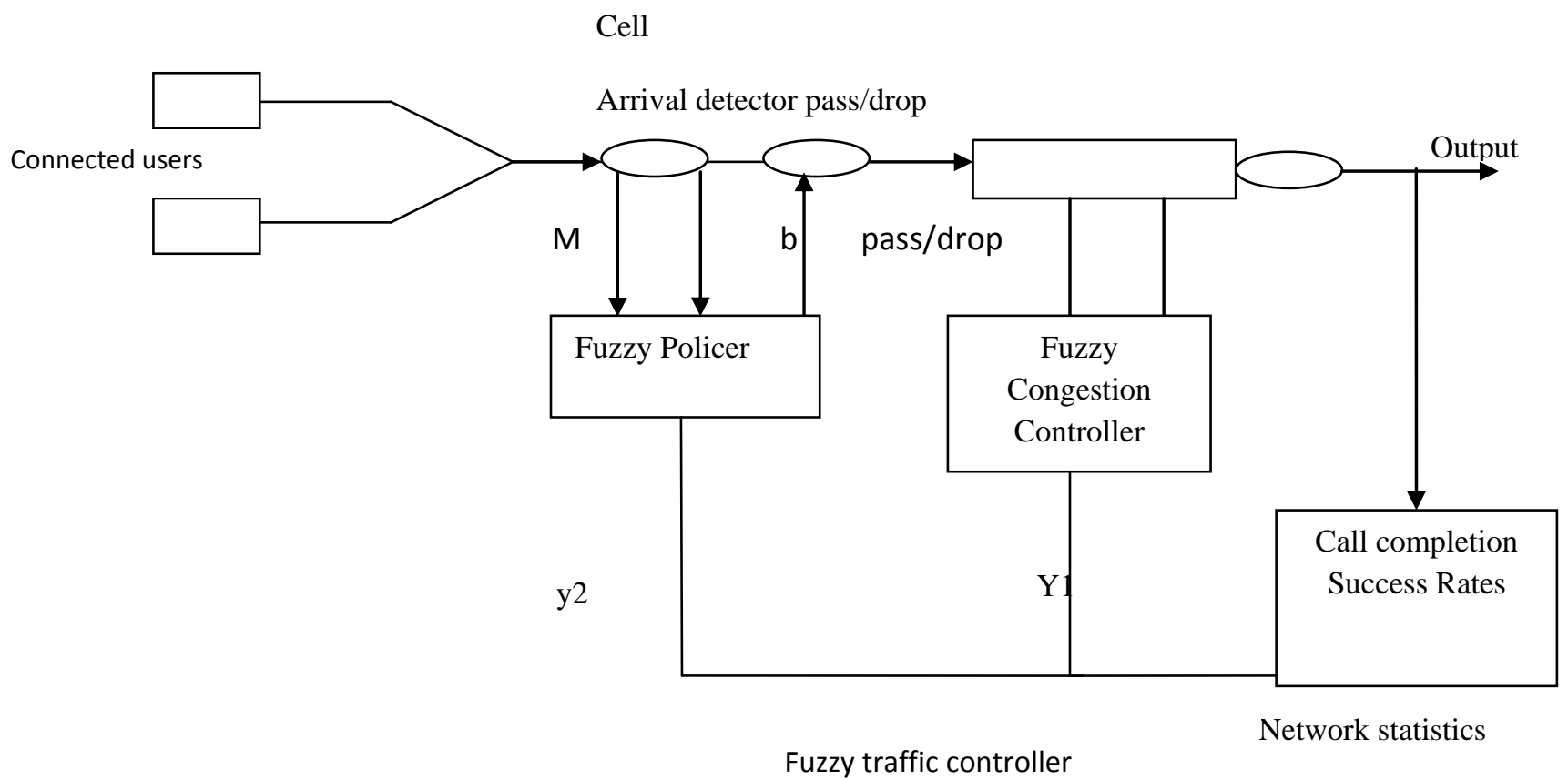

Figure: 2 Architectural design of Proposed System

Table 1: Congestion Load Environments

\begin{tabular}{|c|c|c|c|c|c|}
\hline Traffic load environments & Mean_Bit_Rate & Mean_Burst_L & Network_S & Retianability & OUT_PUT \\
\hline congestion load environment1 & 1.1 & 1.1 & 0.8989 & 0.9 & 0.5 \\
\hline congestion load environment2 & 1.2 & 1.2 & 0.819 & 0.9222 & 0.5 \\
\hline congestion load environment3 & 1.3 & 1.3 & 0.819 & 0.9222 & 0.761 \\
\hline congestion load environment4 & 1.4 & 1.4 & 0.94 & 0.9222 & 0.99 \\
\hline congestion load environment5 & 1.4 & 1.4 & 0.819 & -0.0333 & 0.5 \\
\hline
\end{tabular}

From table 1, it was observed that the effect of variations of call retainability on congestion control in load environments 4 has the value of mean bit rate (1.4), mean burst (1.4), state of the network (0.94) which is positive and retainability (0.9222) and output signal to the service rate is 0.99 , this implies that the service rate should be free for calls to flow. But the variation at congestion load environments five of retainability is $(-0.03)$ it implies that when it is negative, it sends signal to the service rate capacity (queue capacity) to compress incoming calls rates, (that is limiting the call connections) thus forming queue to delay connection of calls, until the network congestion is sorted out. The effect of

network variation on congestion control was represented graphically in figure 3.The graphical representation has call loss ratio at horizontal axis(y- axis) and call retainability at vertical axis (x-axis) in call loss ratio at 0.9 there will be no congestion the network state is free, showing that the system are not congested at the moment but at a point when the performance of network move from 0.9 to 0.5 , it shows that at 0.5 the system is congested at that moment and calls cannot flow very well.

\subsection{Input Design}

The input design figure 4 consists of four-input boxes such as queue length, rate of change of queue, state of network and networks retainablilty. It has a submit button to submit the inputs to the system. The four inputs can be represented with codes as follows: comply, sort of comply, positive or negative and staying network.

\subsection{Output Design}

The output design figure 5 consists of network state, network retainability and control decision. The two outputs network

state and network retainability are re-used to stabilize the system. From the output the state of the network is positive showing that the system is congestion free and control decision will pass all calls successfully without distortion. 


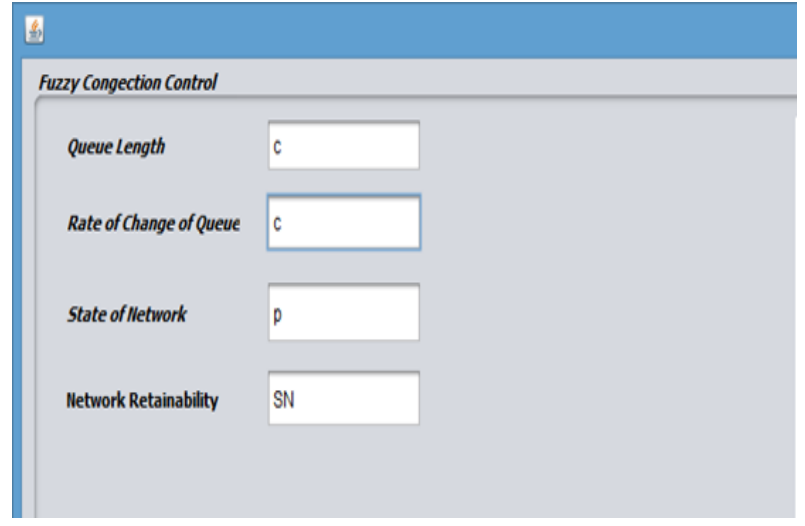

Figure 4: Input Design

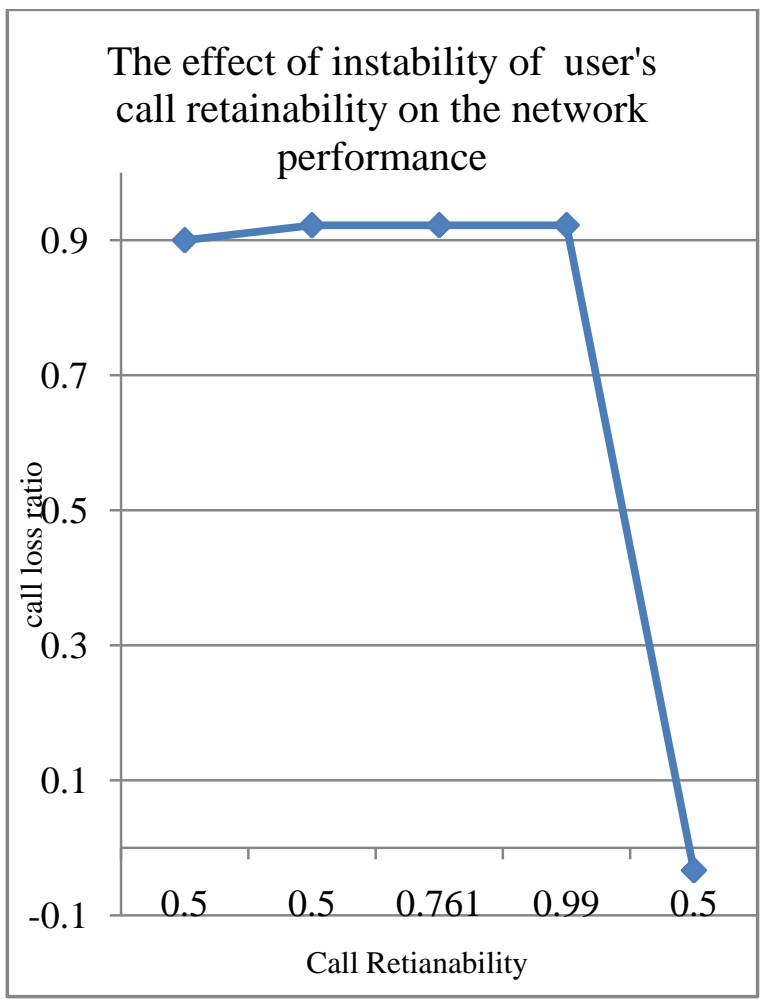

Figure 3: Graphical representation of network variation

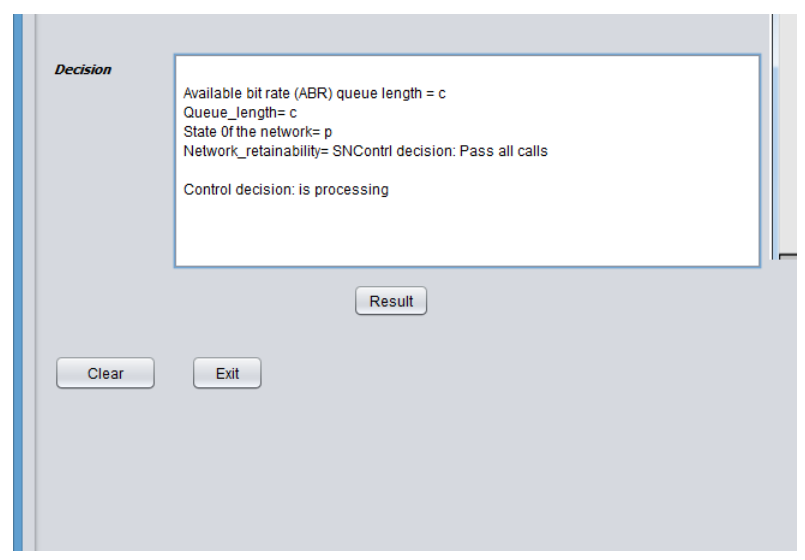

Figure 5: Output design

\section{SIMULATION RESULT}

The simulation result figure 6 shows the four inputs in the architectural system that monitor the network system. The mean bit- rate, mean- burst- rate, network state and call completion success rate that gave output to control the network load traffic. The mean-bit-rate and mean- burst rate are connected from the fuzzy policer. When the user send the call, the cell arrival detector, detect the call at certain point whereby the call capacity are more than the network, the fuzzy policer will alert the fuzzy logic controller to adjust its current transmission rate. From the simulation result, the burst rate shows when calls are more than network capacity. In figure 5 , it shows that the mean- bit-rate is 1.1 , mean-burst length is 1.1 , network state is 0.894 , call completion success rate is 0.893 and queue length is 0.5 and the output is 0.5 . The output produced is positive, this shows that the system is free meaning that all calls can pass without distortion.

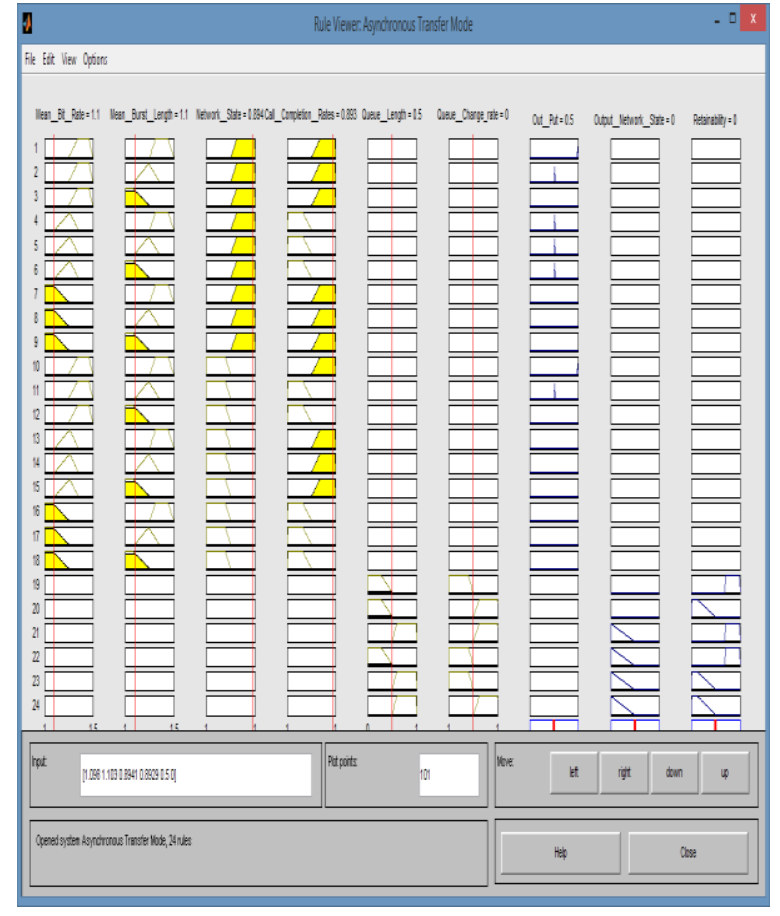

Figure 6: Simulation result

\section{CONCLUSION}

Congestion is serious issue encountered by every network users in day to day activities. As such there is need to develop a system that can reduce congestion. In this work, Call completion success rate is an additional parameter that was introduced to the existing system to reduce congestion in ATM network. Call completion success rate established how users made call successfully without distortion of network.

\section{REFERENCES}

[1] Ding, W. Marchionini, G.I. and Prycker M. 1996. Asynchronous Transfer Mode, solutions for Broadband ISDN, 2nd edn. Ellis Horwood,

[2] Salim Hariri and Bei Lu 1996. ATM-Based Parallel and Distributed Computing

[3] Açar, G. and Rosenberg, C. 2001. Weighted Fair Bandwidth-on-Demand (WFBoD) for Geostationary

[4] Kuboye, 2010. Data \& Computer Communications 6th. ed., Prentice Hall, Networks with On-Board Processing Computer Networks

[5] Syski .C. and Mazumdar, R. 1999. A note on the conservation law for continuous reflected processes and 
its application to queues with fluid inputs Queueing Systems

[6] Chennai , Ferro, F. Potorti, and Maral, G. 2012. Delay Analysis for Inter-LAN traffic using two suitable TDMA satellite access schemes. International Journal of Satellite Communication

[7] Andreas Pitisillides and Ahmet Sekerciouglu 1997. Fuzzy Logic based Congestion Control http://Citeseerx.ist.psu.edu/viewdoc/

[8] J. Zrida, A. Benzaouia, F. Mesquine and S. El Faiz 2003. Rate-Based Flow Fuzzy Controller for Communication Systems. Proceedings of 1st African Control Conference, Cape Town, South Africa.

[9] Hung , R. 1998. Asynchronous Transfer Mode Networks Performance Issues, Artech House

[10] C. Chrysostomou, A. Pitsillides, Y. A. Sekercioglu 2009. Fuzzy Explicit Marking: A Unified Congestion
Controller for Best-Effort and Diff- Serv Networks Journal on Computer Networks

[11] O.P.Lim, T.C. Ling, K.K.Phang, 1996. Development of fuzzy logic control systems. Malaysian journal of computer science.

[12] ImanAskerbeyli and FidanAybikeGedik 2001. NeuroFuzzy Approach for solving Communication Network Proceedings of IEEE International Fuzzy Systems Conference, Problems" International Journal of Electric and Computer Science (IJECS),

[13] Weng Tat Lau, K.K. Phang, MashkuriYaacob. 1999. Managing bandwidth in ATM networks with bursty traffic, IEEE Network

[14] The ATM Forum, 1998. ATM User-Network Interface (UNI) Signalling Specification, Version 3.1 